reasonably be interpreted as equal interference levels for all. It is essential that the ITU modernize and improve its protection standards for the passive services as it introduces and regulates existing and new space-based telecommunications industries.

2. Limits of Unwanted Emissions-Unwanted emissions from other spectrum users in adjacent or far-removed bands have become a limiting factor for the use of many passive bands. Passive users are intend on lowering the limits for out-of-band and spurious emissions. Although the protection of the passive services was one of its principal objectives, ITU-R Task Group 1-3 on "Spurious Emissions" has recommended standards for WRC-97 that do little to protect the passive services. Continued efforts within a new Task Group 1-5 are required to bring the standards for unwanted emissions in line with current radio technology.

3. Space Services-The explosive growth of the use of satellites by various services offers great benefits to mankind yet the current growth in number poses an ominous threat for scientific spectrum users. Downlink emissions can be particularly damaging for ground-based astronomical telescopes and already the emissions of GLONASS and ASTRA-1D have severely curtailed observations. The new IRIDIUM system poses a similar threat. In building space vehicles the highest engineering standards should not be sacrificed in favor of economic arguments. In particular, electro-magnetic environmental impact statements and extensive testing must be required before launching space-borne transmitter systems.

4. Spectrum Bands Above $30 \mathrm{GHz}$-The increasing commercial use of frequency bands above $30 \mathrm{GHz}$ poses new problems for the scientific spectrum users. In particular, the protection of spectral neighbors will require higher engineering standards and vastly improved filter technology. Regulations for high frequency bands up to and beyond $1000 \mathrm{GHz}$ should take into account the lessons learned at lower frequencies to prevent similar shortcomings.

5. Spectrum Splintering-The ITU Allocation Table contains spectrum designations for many "unlike" services with different operational standards in adjacent bands. Many of these bands are rather narrow and extensive coordination is required for these users to operate together and not interfere. Even today about seventy percent of all radio astronomy bands are threatened by satellite links and still new satellite downlink allocations are sought next to passive bands. Administrations need to strive to allocate "like" services to broader band sections and avoid further splintering of the spectrum in increasingly narrow bands.

6. Sharing Considerations-Creative time sharing and geographical sharing schemes may provide limited access to certain bands, which are allocated to other services but have scientific potential. Geographical sharing schemes will require the creation of coordination zones or quiet zones by national administrations to provide local protection from ground-based services for sensitive observatories.

Peaceful coexistence in the spectrum for passive and active services is possible and depends on mutual respect for each other's activities. As spectrum occupation increases, the need to protect all passive and active spectrum users becomes more urgent. Only by mutual understanding and cooperation can we keep the radio windows to the Universe open and clean and in reserve for passive use by future generations.

\title{
11. THE SPACE DEBRIS ENVIRONMENT OF THE EARTH, AMOUNTS AND GROWTH
}

\section{W. Flury (ESA/ESOC)}

Since 1957, more than 3800 launches have led to more than 8000 trackable objects currently in orbit around the Earth. Surveillance, that is detecting and tracking space objects with radar, optical and infra-red sensors, is carried out by the space surveillance networks of the United States and Russia. The trackable objects have typically a minimum size of about $10 \mathrm{~cm}$ at low altitude and about $1 \mathrm{~m}$ at the geostationary orbit. Of the large number of catalogued objects fewer than about 500 are operational satellites. The remainder are abandoned satellites (21\%), upper stages (16\%), fragments of satellites and upper stages (45\%), and mission-related objects (12\%) such as lens covers, separation bolts or clamp-bands. A much larger number of smaller objectsunobservable by routine space surveillance-maybe 100,000 to 150,000 larger than $1 \mathrm{~cm}$ and thus capable of damaging a spacecraft, is in orbit. With special campaigns of powerful radar facilities, 
e.g. Haystack, FGAN, or the combined use of FGAN with the $100 \mathrm{~m}$ Effelsberg radio telescope, statistical information on $1 \mathrm{~cm}$ size objects in Low-Earth orbit (LEO) can be gained.

With an uncontrolled growth orbital debris could become a major hazard for all human activities in space. Already now orbital debris is a potential risk in two important regions, namely at low Earth altitude, and in the geostationary ring. An indication of the degrading quality of the space environment are the first confirmed collision of an operational spacecraft (Cerise) with a rocket fragment, avoidance manoeuvres of the US Space Shuttle and of unmanned spacecraft in LEO, and the more than 60 replacements of windows of the US Space Shuttle damaged by impacts of small-size debris and meteoroids. Space debris is also degrading astronomical observations as large scale exposures of the sky may contain satellite trails.

This presentation will provide an overview of the terrestrial space debris environment. Sources for the catalogued objects and small-size space debris will be reviewed. Models for the spatial distribution will be presented. Explosions and collisions as the main debris sources will be addressed. Finally, the long-term evolution and debris reduction measures will be reviewed.

\section{ENVIRONMENTAL DISTURBANCES OF ASTRONOMICAL OBSERVATIONS}

\section{J. Kovalevsky (CERGA, Observatoire de la Côte d'Azur)}

This presentation is aimed at describing several effects, generally of geophysical origin, which contribute, in addition to sky glow or electromagnetic wave interferences, to degrade astronomical observations from the ground.

The efficiency of an astronomical instrument must be considered together with all the atmospheric layers crossed by the incoming light. This optically active element affects the shape of the image and the apparent direction of the observed celestial body. Astrometric measurements may be significantly biased by un-modelled spurious refraction effects. But the most important source of disturbances is the local atmospheric turbulence which is the major factor of astronomical seeing. The height of the boundary layer is function of the existence of heat sources. In day-time, it is governed by the Sun, but in night-time, the presence of buildings, roads, vegetation of various types, has a definite influence on the quality of the atmospheric images, increasing their unstability and introducing sometimes an inclination of the atmospheric layers, causing abnormal refraction.

The chemical composition of the atmosphere has evidently a direct effect on spectroscopy. This is particularly important in millimeter and submillimeter band astronomy, in the research of various interstellar or circumstellar molecules. The atmospheric emissions and opacity in this wavelength domain are major perturbations in recognizing the spectral lines of these extraterrestrial cmissions. Increase in opacity may also occur after major geophysical events such as aerosols or dust from volcanos.

Optical interferometres are very sensitive to any perturbation of the optical path lengths. This can be produced by deformations of the wavefront due to turbulence, and particularly long period deformations in the higher atmospheric layers. Another cause is the variation of the baseline, consequence of seismic activity. Very significant perturbations are produced by oceanic waves which and are important even several hundreds of kilometers away. Storms on the ocean may be sources of important deterioration of interferometric observations.

Some of the atmospheric dynamical effects may be compensated using active or arlaptative optics. It has however to be noted that these techniques correct the wavefront only within a few arc seconds. This is certainly the solution when a single star is being observed, but does not apply for larger field studies. It is also conceivable that is the future, interferometric baselines could be corrected in real time from seismic simultaneous readings. Millimetric and submillimetric observations can get rid of a large part of spectroscopic pollution in hight altitude sites. But most of the ground based astronomical observations will continue to be lighly sensitive to these environmental perturbations.

\section{THE AVOIDANCE OF MAN-MADE POLLUTION IN INTERPLANETARY SPACE}

C. S. L. Keay (University of Newcastle)

At the 20th General Assembly of the International Astronomical Union in Baltimore, U.S.A., in 1988 members of Commission 22 and several Commissions in what is now the Division of Plan- 